\title{
On the environments and progenitors of supernova remnants associated with highly magnetized neutron stars
}

\author{
S. Safi-Harb $\dagger$ and H. S. Kumar \\ Department of Physics \& Astronomy, University of Manitoba, Winnipeg, Canada \\ email: samar@physics.umanitoba.ca
}

\begin{abstract}
The distinction between the high-magnetic field pulsars (HBPs, thought to be mainly rotation-powered) and magnetars (commonly believed to be powered by their super-strong magnetic fields) has been recently blurred with the discovery of magnetar-like activity from the HBP J1846-0258 in the SNR Kes 75. What determines the spin properties of a neutron star at birth and its manifestation as a magnetar-like or more classical pulsar is still not clear. Furthermore, although a few studies have suggested very massive progenitors for magnetars, there is currently no consensus on the progenitors of these objects. To address these questions, we examine their environments by studying or revisiting their securely associated SNRs. Our approach is to: 1) infer the mass of their progenitor stars through X-ray spectroscopic studies of the thermally emitting supernova ejecta, and 2) investigate the physical properties of their hosting SNRs and ambient conditions. We here highlight our detailed studies of two SNRs: G292.2-0.5, associated with the HBP J1119-6127, and Kes 73, associated with the AXP 1E 1841-045, and summarize the current view of the other (handful) HBP/magnetar-SNR associations.
\end{abstract}

Keywords. (ISM:) supernova remnants; stars: neutron; (stars:) pulsars: individual (J11196127, 1E 1841-045); X-rays: ISM, X-rays: individual (G292.2-0.5, Kes 73)

\section{High magnetic field pulsars and magnetars: On their link, progenitors, and association with supernova remnants}

The past decade has witnessed a synergy of X-ray and radio observations revealing a diversity of young isolated neutron stars with magnetic fields spanning five orders of magnitude. These include the magnetars (the anomalous X-ray pulsars, AXPs; and the soft gamma-ray repeaters, SGRs), high-magnetic field radio pulsars (HBPs), Rotating Radio Transients, and the Central Compact Objects in Supernova Remnants (SNRs), in addition to the more 'classical' rotation-powered pulsars like the Crab. X-ray observations of the HBP J1846-0258 in the SNR Kes 75 showed the first evidence of a magnetarlike behaviour from a Crab-like pulsar, suggesting that the class of HBPs is linked to magnetars (Kumar \& Safi-Harb 2008, Gavriil et al. 2008). More recently, a magnetar was discovered with a relatively low magnetic field (Rea et al. 2010), a surprising result questioning the need for a high dipolar field $\left(B \geqslant B_{Q E D}=4.4 \times 10^{13} \mathrm{G}\right)$ for magnetar-like activity. This also suggests that these objects are more common than generally believed and that there is likely an evolutionary link between the different classes of neutron stars.

While observationally there is so far no consensus on magnetar progenitors, there is accumulating evidence for them originating from very massive progenitors $\left(M>30 M_{\odot}\right)$, as inferred for SGR 1806-20 and CXOU J164710.2-455216, associated with very massive clusters (Figer et al. 2005, Muno et al. 2006), the presence of a stellar-wind blown

\section{$\dagger$ Canada Research Chair}


bubble around AXP 1E 1048.1-5937 (Gaensler et al. 2005), and the Wolf-Rayet progenitor inferred for Kes 75 hosting HBP J1846-0258 (Morton et al. 2007). However a lower progenitor mass of $17 M_{\odot}$ has been suggested for SGR 1900+14 (Davies et al. 2009).

To further address the link between HBPs and magnetars and their progenitors, we investigate their associated SNRs in X-rays. X-ray spectroscopy provides a powerful tool to infer the mass of the progenitor. This is achieved by fitting the X-ray spectra of young, ejecta-dominated, SNRs and comparing the fitted metal abundances to nucleosynthesis model yields. Furthermore, the parameters inferred from fitting the X-ray spectrum of the blast wave yield other intrinsic properties of the SNR (explosion energy, age, ambient density), thus shedding light on their environment and evolutionary stage.

Currently, there is only a handful of associations: two SNRs securely associated with HBPs: G292.2-0.5/J1119-6127 and Kes 75/J1846-0258, and four SNRs associated with magnetars: Kes 73/1E 1841-045, CTB 109/1E 2259+586, G327.24-0.13/1E 1547.0-5408, and G337.0-0.1/SGR 1627-41. Other proposed associations are: W41/magnetar Swift J1834.9-0846, G29.6+0.1 and CTB 37B with magnetars candidates AX J1845-0258 and CXOU J171405.7-381031, respectively, the candidate SNR G333.9+0.0/radio magnetar PSR J1622-4950, G42.8+0.6/SGR 1900+14, G353.6-0.7/magnetar candidate XMMU J173203.3-344518, and N49/SGR 0526-66 in the LMC $\dagger$. We here briefly summarize our dedicated studies of G292.2-0.5 and Kes 73 (Kumar et al. 2012a, b), and conclude with a summary of the properties of their associated SNRs studied in X-rays.

\section{The SNRs G292.2-0.5 and Kes 73}

The SNR G292.2-0.5 is associated with the HBP J1119-6127 which has a rotation period $P \sim 408 \mathrm{~ms}$, a characteristic age $\tau \sim 1.6 \mathrm{kyr}$ (with an upper limit on its age of $1.9 \mathrm{kyr}$ ), and a dipole magnetic field $B \sim 4.1 \times 10^{13} \mathrm{G}$. The combined Chandra and XMMNewton study shows that the plasma is best described by a two-component thermal+nonthermal model. The thermal component is fitted with a non-equilibrium ionizationmodel with a high temperature $k T$ ranging from $1.3 \mathrm{keV}$ in the western side to $2.3 \mathrm{keV}$ in the east, a column density increasing from $1.0 \times 10^{22} \mathrm{~cm}^{22}$ in the west to $1.8 \times 10^{22} \mathrm{~cm}^{-2}$ in the east, and a low ionization timescale ranging from $5.7 \times 10^{9} \mathrm{~cm}^{-3} \mathrm{~s}$ in the SNR interior to $3.6 \times 10^{10} \mathrm{~cm}^{-3} \mathrm{~s}$ in the western side. An additional hard non-thermal component for the eastern and western sides of the pulsar can be partly attributed to leakage of relativistic particles from the pulsar or its associated nebula. The spatial and spectral differences across the SNR are consistent with the presence of a dark cloud in the eastern part of the SNR absorbing the soft X-ray emission. The metal abundances inferred for the western side of the SNR are consistent with solar or sub-solar values, characterizing the emission from the supernova blast wave; while the interior regions indicate the presence of slightly enhanced abundances from $\mathrm{Ne}, \mathrm{Mg}$, Si, hinting for the first time at the presence of reverse-shocked ejecta. We infer a high progenitor mass of $\sim 30 M_{\odot}$ suggesting a type $\mathrm{Ib} / \mathrm{c}$ supernova, an SNR age of $\sim 4-7 \mathrm{kyr}$, and a low ambient density (Table 1 ). The discrepancy between the SNR and pulsar's age can be attributed to a variable braking index for the pulsar which recently showed unusual timing characteristics in the radio.

For the SNR Kes 73 associated with AXP 1E 1841-045 (P 11.8 s, $\tau \sim 4.7 \mathrm{kyr}, B \sim 7 \times$ $10^{14} \mathrm{G}$ ), our Chandra and XMM-Newton spatially resolved spectroscopic study requires a two-component non-equilibrium ionization thermal model. The soft-component has temperatures $\sim 0.3-0.5 \mathrm{keV}$ and ionization timescales $\gtrsim 10^{12} \mathrm{~cm}^{-3} \mathrm{~s}$ with enhanced metal

$\dagger$ see http://www.physics.mcgill.ca/ pulsar/magnetar/main.html for the magnetars catalogue, and http://www.physics.umanitoba.ca/snr/SNRcat for the Galactic SNRs high-energy catalogue, associations with compact objects, and references not listed here due to space limits. 
Table 1. SNR-HBP/magnetars associations with SNR properties inferred from X-ray studies.

\begin{tabular}{llllllr}
\hline SNR/PSR & $D(\mathrm{kpc})$ & $n_{0}\left(\mathrm{~cm}^{-3}\right)$ & $v\left(\mathrm{~km} \mathrm{~s}^{-1}\right)$ & Age $(\mathrm{kyr})$ & $E_{0}\left(10^{51}\right.$ ergs $)$ & Ref. \\
\hline G292.2-0.5/J1119-6127 & 8.4 & $0.02 f^{-1 / 2}$ & 1100 & $4.2-7.1$ & $0.6 f^{-1 / 2}$ & {$[1]$} \\
Kes 75/J1846-0258 & 10.6 & $0.2-1.2$ & 3,700 & $0.6-0.9$ & - & {$[2]$} \\
\hline Kes 73/1E 1841-045 & 8.5 & $(0.3-0.9) f^{-1 / 2}$ & 1000 & $1.1-2.1$ & $0.2 f^{-1 / 2}$ & {$[3]$} \\
CTB 109/1E 2259+586 & 3.0 & 0.16 & $720-1140$ & $7.9-9.7$ & $0.7-1.8$ & {$[4]$} \\
CTB 37B/J1714-3810 & 10.2 & $0.2-0.4$ & - & $0.4-3.1$ & - & {$[5]$} \\
N49/SGR 0526-66 & 50 & $1.9 f^{-1 / 2}$ & 700 & 4.8 & $1.8 f^{-1 / 2}$ & {$[6]$} \\
\hline
\end{tabular}

Notes: $f$ is the volume filling factor. The lower and upper age estimates for G292.2-0.5 and Kes 73 correspond to the free expansion and Sedov phases, respectively. For the other SNRs, the age was mainly estimated assuming the Sedov phase (for CTB 37B using the ionization timescale). References: [1] Kumar et al. 2012a, [2] Temim et al. 2012, Su et al. 2009, [3] Kumar et al. 2012b, [4] Sasaki et al. 2004, [5] Nakamura et al. 2009, [6] Park et al. 2012.

abundances; while the hard-component exhibits plasma temperatures $\sim 1.1-1.7 \mathrm{keV}$ and low ionization timescales $\sim(0.5-2.8) \times 10^{11} \mathrm{~cm}^{-3} \mathrm{~s}$ with solar abundances. These results indicate that the soft-component arises from the reverse-shocked ejecta with most regions showing plasma that has reached ionization equilibrium, while the hard-component originates from the blast wave shocking an ambient medium with an average density of $n_{0} \sim 0.5 \mathrm{~cm}^{-3}$. We infer a progenitor mass of $\sim(25-30) M_{\odot}$, supporting the earlier prediction of a SN type IIL/b for the remnant, and an SNR age of $\leqslant 2.1$ kyr (Table 1 ).

\section{Summary}

In summary, most current studies point to highly magnetized neutron stars originating from very massive progenitors. Table 1 highlights the SNR properties of the X-ray studied associations. These results also suggest young SNRs (age $<10 \mathrm{kyr}$ ) expanding in a relatively low-density medium for the Galactic SNRs. Further dedicated studies in $\mathrm{X}$-rays and other wavelengths, as well as improved nucleosynthesis models, are needed to confirm these calculations and increase the sample of neutron star-SNR associations. S.S.-H. acknowledges the support of the CRC program, NSERC, CFI, CITA, and CSA.

\section{References}

Davies, B., et al. 2009, ApJ, 707, 844.

Figer, D. F., et al. 2005, ApJ, 622, L49.

Gaensler, B. M., et al. 2005, ApJ, 620, 95.

Gavriil, F., et al. 2008, Science, 319, 1802.

Kumar, H. S. \& Safi-Harb, S. 2008, ApJL, 678, L43.

Kumar, H. S., Safi-Harb, S., \& Gonzalez, M. E. 2012a, ApJ, 754, 96.

Kumar, H. S., Safi-Harb, S., Slane, P. O., \& Gotthelf, E. V. 2012b, to be submitted to ApJ.

Morton, T. D., et al. 2007, ApJ, 667, 219.

Muno, M. P., et al. 2006, ApJ, 636, 41.

Nakamura, R., et al. 2009, PASJ, 61, 197.

Park, S., et al. 2012, ApJ, 748, 117.

Rea, N., et al. 2010, Science, 330, 944.

Sasaki, M., et al. 2004, ApJ, 617, 322.

$\mathrm{Su}$, Y., et al. 2009, ApJ, 694, 376.

Temim, T., Slane, P. O., Arendt, R. G., \& Dwek, E. 2012, ApJ, 745, 46. 